\title{
Liraglutide-induced reduction of myocardial ischemia- reperfusion injury in rats via ERK1/2 signaling pathway
}

\author{
Zhao-ying Liu ${ }^{1}$, Qing-rong $\mathrm{Ji}^{1}$, Shun-peng $\mathrm{Hu}^{{ }^{1}}$, Dong-hao Zhou ${ }^{2}$, Gui-ling Sun ${ }^{1}$ \\ and Pin-jun Zhu ${ }^{1}$ \\ ${ }_{1}^{1}$ Department of Cardiology, ${ }^{2}$ Department of Endocrinology, Linyi People's Hospital in Shandong Province, Linyi City, Shandong \\ Province, 276000, China
}

${ }^{*}$ For correspondence: Email: hushunpeng309@126.com; Tel: 0539-8078080

Sent for review: 2 May 2017

Revised accepted: 28 July 2017

\begin{abstract}
Purpose: To investigate the protective effect of liraglutide on myocardial ischemia reperfusion (I/R) injury and its molecular mechanism.

Methods: Ischemia reperfusion model male Sprague-Dawley (SD) rats were randomly divided into negative control group, I/R group (saline), liraglutide group (liraglutide) and PD group (liraglutide + PD98059). The weight of myocardium in ischemic and infarction areas of the heart, myocardial injury biomarker, oxidative stress, as well as expressions of mRNA molecules of apoptosis were determined.

Results: The myocardial mass of ischemic and infarcted areas of the heart (relative to left ventricular mass) of $I / R$ group were significantly higher $(p<0.05)$ than those of negative control group, but significantly lower in liraglutide group than in $1 / R$ group $(p>0.05)$. However, the parameters were significantly higher in PD group than in liraglutide group $(p<0.05)$. CK, CK-MB and LDH activities, as well as levels of cTnl and CTnT in I/R group were significantly higher $(p<0.05)$ than those of negative control group. However, the parameters were significantly lower $(p<0.05)$ in liraglutide group than in $I / R$ group, but higher in PD group $(p<0.05)$ than in liraglutide group. Serum SOD, GSH-PX, CAT activities and $t B C l-2$ mRNA expression were significantly lower in $1 / R$ group than those of negative control group $(p<0.001)$, while those PD group were significantly lower than those of liraglutide group $(p<0.001)$.

Conclusion: Liraglutide alleviates myocardial ischemia-reperfusion injury and inhibits oxidative stress and apoptosis via ERK1/2 signaling pathway in rats, but further studies are required to ascertain the clinical efficacy and safety of the compound.
\end{abstract}

Keywords: Ischemia-reperfusion injury, Liraglutide, ERK1/2 signal pathway, Oxidative stress, Apoptosis

Tropical Journal of Pharmaceutical Research is indexed by Science Citation Index (SciSearch), Scopus, International Pharmaceutical Abstract, Chemical Abstracts, Embase, Index Copernicus, EBSCO, African Index Medicus, JournalSeek, Journal Citation Reports/Science Edition, Directory of Open Access Journals (DOAJ), African Journal Online, Bioline International, Open-J-Gate and Pharmacy Abstracts

\section{INTRODUCTION}

Myocardial ischemia reperfusion (IR) injury refers to the pathophysiological process of aggravated injury after ischemic myocardial perfusion. In clinical practice, IR is an important factor that affects myocardial infarction thrombolysis. Therefore, reduction of myocardial IR injury is an important objective in improvement of reperfusion in patients with myocardial infarction
[1-3]. Liraglutide is a new drug that has been used for type 2 diabetes in recent years. Pharmacologically, it is a glucagon-like peptide I (GLP-1) analogue, which effectively reduces blood glucose levels, regulates blood lipid metabolism and relieves insulin resistance in Type 2 diabetes. Recent studies have reported that liraglutide not only regulates glucolipid metabolism, but also improves endothelial and myocardial cell functions. It also reduces 
myocardial cell injury caused by hypoxia and hypoxia re-oxygenation conditions $[4,5]$.

The aim of this study was to study the protective effect of liraglutide on myocardial ischemiareperfusion injury in an animal model as well as the molecular mechanisms involved.

\section{EXPERIMENTAL}

\section{Materials}

Adult male SD rats (weighing 240 - $300 \mathrm{~g}$ ) used in this study were provided by the Animal Experimental Center of Shandong University. Liraglutide injection $(6 \mathrm{mg} / \mathrm{mL})$ was product of Novo Nordisk Pharmaceutical Co., Ltd, China. The narcotics, surgical instruments and small animal ventilator for animal experiments were supplied by the Animal Experiment Center of Shandong University; electrochemiluminescence kits and radioimmunoprecipitation kits were provided by Nanjing Jiancheng Bioengineering Institute of China, RNA extraction kits, cDNA first-strand synthesis kits and fluorescence quantitative PCR kits were purchased from Beijing Tiangen Biochemical Company, China. PCR primers were obtained from Shanghai Sangon Company.

\section{Animal groups and treatments}

$S D$ rats were randomly divided into negative control, I/R, liraglutide and PD groups (6 rats per group). The $\mathrm{I} / \mathrm{R}$, liraglutide and $\mathrm{PD}$ groups were made into myocardial ischemia-reperfusion injury models according to the following procedure. The rats were anaesthetized by intraperitoneal injection of chloral hydrate and placed on the operating table in supine position. Median neck incision was made, the trachea was separated and intubated, and a small animal ventilator was connected.

Thereafter the electrode was placed on the left lower extremity for continuous recording of electrocardiogram. An incision was made between the $3^{\text {rd }}$ and $4^{\text {th }}$ ribs to expose the heart, and the left anterior descending branch of the coronary artery was isolated and clamped with bulldog clamp for 40 min. ST elevation and peak $\mathrm{T}$ wave in the electrocardiogram indicated evidence of myocardial infarction. Then the bulldog clamp was loosened, and myocardial perfusion was restored for $4 \mathrm{~h}$ for completing establishment of myocardial I/R model. The negative control group received only model operation in which the left anterior descending branch of the coronary artery was separated after anesthesia, but without clamping.

\section{Drug treatments}

Eight days before the model making, drug intervention was done for 7 continuous days, and myocardial I/R injury model was established on the $8^{\text {th }}$ day. In the drug intervention procedure, the liraglutide group received $0.07 \mathrm{mg} / \mathrm{kg}$ of the drug once a day by subcutaneous injection. The PD group received the same dose of liraglutide once a day subcutaneously in addition to 1 $\mathrm{mg} / \mathrm{kg}$ of PD98059 also once a day. The negative control and $\mathrm{I} / \mathrm{R}$ groups received an equivalent volume of saline subcutaneously once a day.

\section{Determination of parameters related to myocardial ischemia and infarction}

Four hours after myocardial blood reperfusion, the left anterior descending branch of the coronary artery was blocked, and $1 \mathrm{~mL}$ of $5 \%$ Evans blue was injected via the internal jugular vein. Thereafter the heart was excised the left ventricle and was separated and frozen in liquid nitrogen for $10 \mathrm{~min}$. The left ventricular myocardial tissue was cut into $2 \mathrm{~mm}$-thick sections and examined under the microscope for detailed evaluation of hypoxia-induced changes. Areas stained blue were considered normal myocardium, while the areas stained red were ischemic, non-infarction myocardium. The graystained areas were indicative of infracted myocardium. Electronic balances were used to weigh the myocardial mass of the ischemic and infarction areas, and the percentages of myocardial mass of ischemic and infarction areas relative to left ventricular mass were calculated.

\section{Assay of biochemical indices}

Peripheral blood specimens were collected $4 \mathrm{~h}$ after myocardial blood reperfusion, allowed to clot and centrifuged get serum samples. Electrochemiluminescence kits were used to determine the levels of creatine kinase (CK), creatine kinase isoenzyme (CK-MB), LDH, cTnl and cTnT. Radio-immunoprecipitation kits were used to determine activities of SOD, GSH-Px and CAT, as well as MDA levels.

\section{Myocardial tissue collection and determina- tion of infarct indices}

Infarcted area of myocardial tissue was weighed, washed twice with saline, and then added to RNA isolation buffer and fully homogenized. RNA extraction kits were used to obtain total RNA in the myocardial tissue, and cDNA first-strand synthesis kits were used to reverse-transcribe the total RNA into cDNA. Thereafter fluorescence 
quantitative PCR kits were used to amplifyBcl-2, Bax, caspase-3, caspase-8 and GAPDH (GAPDH was used as reference). The mean value of the corresponding gene mRNA expression in myocardial tissue of the negative control group was set at 1 to calculate $\mathrm{Bcl}-2$, Bax, caspase- 3 andcaspase- 8 mRNA expression in myocardial tissue of other groups of rats. This research was approved (approval no. is LY2016021) by Animal Ethical Committee of Linyi People's Hospital in Shandong Province according to "Principles of Laboratory Animal Care" (NIH publication no. 85-23, revised 1985) [6].

\section{Statistical analysis}

SPSS version 20 statistical package was used for statistical analyses. Data were analysed for statistical significance using ANOVA. $P<0.05$ was considered to indicate statistically significant difference.

\section{RESULTS}

\section{Myocardial ischemia and infarction}

The results of analysis of mass of ischemic and infarcted areas, and percentage of mass of ischemic and infarction areas relative to left ventricular mass among groups are presented in Table 1. Myocardial mass of ischemic and infarcted areas, and percentages of each of these parameters relative to left ventricular mass of the $\mathrm{l} / \mathrm{R}$ group were significantly higher $(p<$ 0.05 ) than those of negative control group. These parameters were significantly lower in the liraglutide group than in the $\mathrm{I} / \mathrm{R}$ group $(p>0.05)$, but were significantly higher in the PD group when compared with the liraglutide group $(p<$ 0.05).

\section{Myocardial injury indices}

Serum levels of myocardial injury indices - CK, CKM-B and LDH activities as well as CTnl, CTnT, and MDA levels are presented in Table 2. CK, CK-MB and LDH activities, as well as levels of cTnl and cTnT in the I/R group were significantly higher $(p<0.05)$ than those of the negative control group. However these serum parameters were significantly lower $(p<0.05)$ in the liraglutide group than in the $\mathrm{I} / \mathrm{R}$ group, while those of the PD group were significantly higher ( $p$ $<0.05$ ) when compared with the liraglutide group.

\section{Serum levels of oxidative stress indices}

The results of analysis of oxidative stress indices are presented in Table 3. Serum SOD, GSH-Gpx and CAT activities in the $\mathrm{I} / \mathrm{R}$ group were lower, while MDA level of the $\mathrm{I} / \mathrm{R}$ group was significantly higher when compared with corresponding values for the negative control group $(p<0.05)$. However, serum SOD, GSH-Px and CAT activities were significantly higher, while MDA level was significantly lower in the liraglutide group than in the $\mathrm{I} / \mathrm{R}$ group $(p<0.05)$. In the PD group, serum SOD, GSH-Px and CAT activities were significantly lower than those of liraglutide group while MDA level was significantly higher ( $p$ $<0.05)$.

Table 1: Mass of myocardial ischemia and infarction areas of rats (mean $\pm S D, n=6$ )

\begin{tabular}{lcccc}
\hline Group & $\begin{array}{c}\text { Mass of } \\
\text { ischemic area } \\
(\mathbf{m g})\end{array}$ & $\begin{array}{c}\text { Mass of } \\
\text { infarction area } \\
(\mathbf{m g})\end{array}$ & $\begin{array}{c}\text { Ratio of mass of } \\
\text { ischemic area:left } \\
\text { ventricular mass }\end{array}$ & $\begin{array}{c}\text { Ratio of mass of } \\
\text { infarction area:left } \\
\text { ventricular mass }\end{array}$ \\
\hline Negative control & $103.22 \pm 15.58$ & $6.12 \pm 0.93$ & $15.28 \pm 2.96$ & $0.91 \pm 0.12$ \\
I/R & $286.65 \pm 42.35^{\star}$ & $126.59 \pm 16.87^{\star}$ & $48.52 \pm 6.69^{*}$ & $21.43 \pm 4.29^{\star}$ \\
Liraglutide & $175.42 \pm 29.34^{\star}$ & $47.68 \pm 7.95^{\star}$ & $26.91 \pm 4.52^{\star}$ & $7.21 \pm 0.98^{\star}$ \\
PD group & $239.15 \pm 34.61^{\#}$ & $89.49 \pm 12.48^{\#}$ & $37.68 \pm 5.14^{\#}$ & $14.10 \pm 2.16^{\#}$ \\
\hline
\end{tabular}

Compared with negative control group, $p<0.05 ;{ }^{\&}$ compared with I/R group, $p<0.05 ;{ }^{\#}$ compared with liraglutide group, $p<0.05$ values are mean $\pm S D$

Table 2: Myocardial injury indices for rats (mean $\pm S D, n=6$ )

\begin{tabular}{lccccc}
\hline Group & CK (IU/L) & CK-MB (IU/L) & CTnI $(\mu$ g/L) & CTnT (ng/L) & LDH (IU/L) \\
\hline Negative & $22.41 \pm 5.49$ & $46.52 \pm 7.21$ & $0.09 \pm 0.02$ & $32.51 \pm 6.51$ & $53.57 \pm 9.32$ \\
control & & & & \\
l/R & $124.48 \pm 22.39^{*}$ & $193.42 \pm 29.34^{*}$ & $0.69 \pm 0.10^{*}$ & $325.69 \pm 52.67^{*}$ & $254.68 \pm 41.28^{*}$ \\
Liraglutide & $42.16 \pm 7.95^{\star}$ & $93.25 \pm 13.64^{\star}$ & $0.30 \pm 0.06^{*}$ & $125.52 \pm 17.87^{*}$ & $103.59 \pm 15.57^{*}$ \\
PD group & $98.31 \pm 15.57^{\#}$ & $164.22 \pm 23.47^{\#}$ & $0.52 \pm 0.08^{\#}$ & $244.52 \pm 39.61^{\#}$ & $178.55 \pm 25.68^{\#}$ \\
\hline
\end{tabular}


Table 3: Oxidative stress-related levels in rat serum (mean $\pm S D, n=6$ )

\begin{tabular}{lcccc}
\hline Group & SOD (U/L) & GSH-Px (U/L) & CAT (U/L) & MDA ( $\boldsymbol{\mu m o l} / \mathbf{L})$ \\
\hline Negative control & $75.91 \pm 11.35$ & $127.58 \pm 19.75$ & $58.69 \pm 8.54$ & $7.39 \pm 1.27$ \\
I/R & $27.68 \pm 4.51^{*}$ & $42.36 \pm 7.65^{*}$ & $15.23 \pm 1.88^{*}$ & $32.46 \pm 6.58^{*}$ \\
Liraglutide & $62.14 \pm 8.98^{\star}$ & $104.58 \pm 17.52^{\star}$ & $43.57 \pm 6.71^{\star}$ & $14.22 \pm 2.46^{*}$ \\
PD & $35.24 \pm 5.57^{\#}$ & $59.26 \pm 8.14^{\#}$ & $22.67 \pm 3.61^{\#}$ & $26.53 \pm 3.49^{\#}$ \\
\hline
\end{tabular}

"Compared with negative control group, $p<0.05 ;{ }^{8}$ compared with I/R group, $p<0.05 ;{ }^{\#}$ compared with liraglutide group, $p<0.05$

Table 4: Mitochondrial apoptosis-related molecular expression in rat myocardial tissue

\begin{tabular}{lcccc}
\hline Group & Bcl-2 & Bax & Caspase-3 & Caspase-8 \\
\hline Negative control group & $1.00 \pm 0.21$ & $1.00 \pm 0.18$ & $1.00 \pm 0.16$ & $1.00 \pm 0.22$ \\
l/R group & $0.36 \pm 0.06^{*}$ & $2.67 \pm 0.42^{*}$ & $3.02 \pm 0.49^{*}$ & $2.35 \pm 0.38^{*}$ \\
Liraglutide group & $0.89 \pm 0.16^{\star}$ & $1.45 \pm 0.21^{*}$ & $1.52 \pm 0.19^{\star}$ & $1.39 \pm 0.23^{*}$ \\
PD group & $0.44 \pm 0.09^{\#}$ & $2.29 \pm 0.37^{\#}$ & $2.54 \pm 0.41^{\#}$ & $2.04 \pm 0.39^{\#}$
\end{tabular}

"Compared with Negative control group, $p<0.05 ;{ }^{*}$ compared with I/R group, $p<0.05 ;{ }^{\#}$ compared with liraglutide group, $p<0.05$; values are mean \pm SD

\section{Expression of mitochondrial apoptosis- related proteins}

The expression of Bcl-2 mRNA in myocardial tissue of $\mathrm{I} / \mathrm{R}$ group was significantly lower than that in the negative control group, while the expressions of Bax, caspase- 3 and caspase- 8 mRNA expression were significantly higher $(p<$ $0.05)$. Bcl-2 mRNA expression in myocardial tissue of the liraglutide group was significantly higher than that in the $\mathrm{I} / \mathrm{R}$ group while the expressions of Bax, caspase- 3 and caspase- 8 mRNA were significantly lower $(p<0.05)$. The expression of $\mathrm{Bcl}-2$ mRNA in myocardial tissue of the PD group was significantly lower than that of liraglutide group while Bax, caspase- 3 and caspase-8 mRNA expressions were significantly higher $(p<0.05)$. The results are shown in Table 4.

\section{DISCUSSION}

Myocardial ischemia reperfusion (I/R) injury affects the outcome of interventional and thrombolysis therapies after myocardial infarction. Reduction of myocardial I/R injury has been the subject of intensive research in the cardiovascular field [1,3]. Liraglutide, a GLP-1 analogue which regulates glucolipid metabolism, has been increasingly used for the treatment of Type 2 diabetes mellitus and obesity. Recent studies have found that liraglutide not only regulates glucolipid metabolism, but also possesses anti-oxidative stress, anti-apoptosis and cytoprotective effects [7,8]. In order to investigate whether liraglutide has protective effect on myocardial $I / R$ injury in rats, myocardial ischemia and infarcted were analyzed in this study. Analysis of myocardial mass of ischemic and infarction areas showed that the mass of ischemic and infarcted areas, and percentages of myocardial mass of ischemic and infarction areas relative to left ventricular mass of $\mathrm{I} / \mathrm{R}$ group were significantly increased in myocardial tissue of $\mathrm{I} / \mathrm{R}$ group when compared to the negative control group. However, liraglutide intervention significantly reduced these increases, implying that it has protective effect on myocardial $\mathrm{I} / \mathrm{R}$ injury in rats, and can alleviate the myocardial ischemia and infarction caused by $\mathrm{I} / \mathrm{R}$. During $\mathrm{I} / \mathrm{R}$ in myocardial cells, oxidative stress brings about cell damage.

The ROS produced by oxidative stress triggers on myocardial cell rupture, leading to release of myocardial enzymes such as CK-MB, LDH and $\mathrm{CK}$, as well as structural proteins $\mathrm{cTnl}$ and $\mathrm{CTnT}$ into the blood. In the study, analysis of serum levels of some myocardial enzymes and myocardial structural proteins of rats revealed that the activities of $\mathrm{CK}$, CK-MB and $\mathrm{LDH}$, and the levels of cTnl, cTnT and LDH in the I/R group were significantly higher than those of negative control group. Interestingly, the administration of liraglutide significantly reduced the serum activities CK, CK-MB and LDH, as well as CTnl, cTnT levels. This means that liraglutide can inhibit the release of myocardial enzymes and myocardial structural proteins during myocardial $\mathrm{I} / \mathrm{R}$, thereby reducing myocardial $\mathrm{I} / \mathrm{R}$ injury. ROS injures myocardial cells due to peroxidation of membrane lipids, which results in production and release of MDA. Studies have established correlation between MDA levels and the degree of tissue damage arising from oxidative stress $[9,10]$.

The antioxidant enzymes, SOD, GSH-PX and CAT in myocardial cells can remove the reactive oxygen species produced during oxidative stress, 
but the excessive ROS generated during $\mathrm{I} / \mathrm{R}$ overwhelm these enzymes and weaken the body's antioxidant capacity [11,12]. In this study, analysis of the serum levels of MDA and antioxidant enzymes showed that serum SOD, GSH-PX and CAT levels of the I/R group were significantly lower than those of negative control group while MDA level was significantly higher. Again, liraglutide intervention significantly reversed the $\mathrm{I} / \mathrm{R}$-induced decreases in antioxidant enzymes as well as the increases in MDA. This suggests that liraglutide can relieve the myocardial oxidative stress imposed by $\mathrm{I} / \mathrm{R}$, increase the antioxidant capacity, and thus alleviate oxidative stress-induced myocardial I/R injury.

In I/R-induced myocardial injury, the generation of ROS brings about direct damage to cellular structure and function, as well as cell damage through the mitochondrial apoptosis pathway. ROS damage to mitochondria increases mitochondrial membrane permeability to cytochrome $\mathrm{c}$, on entering the cytoplasm, which activates the apoptosis signal cascade mediated by caspase- 8 , and eventually splits the inactive pro-caspase-3 to active caspase-3, thereby causing apoptosis. In the mitochondrial membrane, Bcl-2 and Bax are involved in the control of mitochondrial permeability to cytochrome c. It is known that the mitochondrial permeability of cytochrome $\mathrm{C}$ is reduced when Bcl-2 molecules form homodimers, but is increased when Bax forms heterodimers with $\mathrm{Bcl}-2[13,14]$. Thus the $\mathrm{Bcl}-2 / \mathrm{Bax}$ ratio is a critical factor that regulates mitochondrial apoptosis: decreased the expression of Bcl-2 and increased the expression of Bax result in increases in mitochondrial membrane permeability to cytochrome c, thereby initiating apoptosis through caspase-8/caspase-3 $[15,16]$.

In this study, Bcl-2 mRNA expression in myocardial tissue of $\mathrm{I} / \mathrm{R}$ group was significantly lower than that of the negative control group while Bax, caspase- 3 and caspase- 8 mRNA expressions were significantly higher. This trend was reversed by liraglutide administration, which resulted in increase in the Bcl-2 mRNA expression and decreases in the expressions of Bax, caspase-3 andcaspase-8 mRNAs in myocardial tissue. This means that the liraglutide can relieve myocardial cell damage caused by mitochondrial apoptosis due to myocardial I/R.

ERK $1 / 2$ is an important cellular signaling molecule which is involved in the formation of intracellular mitogen-activated protein kinase signal transduction pathway, and regulation of proliferation, apoptosis, autophagy and oxidative stress in a variety of cells [17-19].

In order to determine whether liraglutide alleviated myocardial $\mathrm{I} / \mathrm{R}$ injury through the ERK1/2 signaling pathway, the ERK1/2-specific inhibitor PD98059 was combined with liraglutide intervention, and differences in myocardial $\mathrm{I} / \mathrm{R}$ injury were compared between liraglutidePD98057 group and the PD group.

The results showed that the masses of ischemic and infarction areas, and their percentages relative to left ventricular mass were significantly higher than corresponding values in the liraglutide-PD98057 group. Moreover, serum CK, CK-MB, cTnl, cTnT, LDH and MDA levels as well as expressions of Bax, caspase- 3 and caspase-8 mRNA in the PD group were significantly higher than corresponding values obtained in the liraglutide-PD98057 group, while serum SOD, GSH-PX and CAT activities as well as Bcl-2 mRNA expression in myocardial tissue were significantly lower. This means that the combination of PD98059 and liraglutide weakened the protective effects of liraglutide on myocardial cell damage, such as alleviation of oxidative stress and inhibition of mitochondrial apoptosis. This finding strongly suggests that the protective effect of liraglutide on myocardial I/R injury was mediated through ERK1/2 signaling pathway.

\section{CONCLUSION}

The results obtained in this study indicate that liraglutide alleviates myocardial ischemiareperfusion injury and inhibit oxidative stress and mitochondrial apoptosis in rats the ERK $1 / 2$ signaling pathway. These findings may provide a new approach for the prevention and treatment on myocardial ischemia and reperfusion injury.

\section{DECLARATIONS}

\section{Acknowledgement}

The authors acknowledge that this study was supported by Centers for Disease Control and Prevention in Laiwu City.

\section{Conflict of Interest}

No conflict of interest associated with this work.

\section{Contribution of Authors}

The authors declare that this work was done by the authors named in this article and all liabilities 
pertaining to claims relating to the content of this article will be borne by them.

\section{Open Access}

This is an Open Access article that uses a funding model which does not charge readers or their institutions for access and distributed under the terms of the Creative Commons Attribution License (http://creativecommons.org/licenses/by/ 4.0) and the Budapest Open Access Initiative (http://www.budapestopenaccessinitiative.org/rea d), which permit unrestricted use, distribution, and reproduction in any medium, provided the original work is properly credited.

\section{REFERENCES}

1. Lakota J. Molecular mechanism of ischemia Reperfusion injury after myocardial infarction and its possible targeted treatment. Int J Cardiol 2016; 220(1): 571-572.

2. Hashmi S, Alsalam S. Acute myocardial infarction and myocardial ischemia-reperfusion injury: a comparison. Int J Clini Exp Pathol 2015; 8(8): 8786-8796.

3. Hollander MR, Waard GAD, Konijnenberg LSF, Putten RMEM, Brom CEVD, Paauw N, Vries HED, Ven PMVD, Aman J, Amerongen GPVN. Correction: Dissecting the Effects of Ischemia and Reperfusion on the Coronary Microcirculation in a Rat Model of Acute Myocardial Infarction. Plos One 2016; 11(7): e0157233.

4. Giglio RV, Patti AM, Nikolic D, Castellino G, Noto $M$, Parrino A, Montalto G, Rizzo M. The extra-glycemic effects of liraglutide: focus on cardiometabolic markers. G Ital Cardiol 2016; 17(4): 253-258.

5. Inoue $T$, Inoguchi $T$, Sonoda $N$, Hendarto $H$, Makimura $H$, Sasaki S, Yokomizo $H$, Fujimura $Y$, Miura $D$, Takayanagi $R$. GLP-1 analog liraglutide protects against cardiac steatosis, oxidative stress and apoptosis in streptozotocin-induced diabetic rats. Atheroscler 2015; 240(1): 250-259.

6. Organization WH. Principles of laboratory animal care. WHO Chron 1985; 39: 51-56.

7. Rizzo $M$, Abate $N$, Chandalia M, Rizvi AA, Giglio RV, Nikolic D, Marino GA, Barbagallo I, Isenovic ER, Banach M. Liraglutide reduces oxidative stress and restores heme oxygenase-1 and ghrelin levels in patients with type 2 diabetes: a prospective pilot study. J Clini Endocrinol Metab 2015; 100(2): jc20142291.

8. Okada K, Kotani K, Yagyu H, Ando A, Osuga J, Ishibashi $S$. Effects of treatment with liraglutide on oxidative stress and cardiac natriuretic peptide levels in patients with type 2 diabetes mellitus. Endocrine 2014; 47(3): 962-964.

9. Halladin NL. Oxidative and inflammatory biomarkers of ischemia and reperfusion injuries. Dan Med J 2015; 61(4): B5054.

10. Tasoulis MK, Douzinas EE. Hypoxemic reperfusion of ischemic states: an alternative approach for the attenuation of oxidative stress mediated reperfusion injury. J Biomed Sci 2016; 23(1): 1-8.

11. Kurian GA, Rajagopal $R$, Vedantham $S$, Rajesh $M$. The Role of Oxidative Stress in Myocardial Ischemia and Reperfusion Injury and Remodeling: Revisited. Oxid Med Cellity 2016; 2016(12): 1-14.

12. Walters JW, Amos D, Ray K, Santanam N. Mitochondrial redox status as a target for cardiovascular disease. Curr Opin Pharmacol 2016; 27(1): 50-55.

13. Schubert C, Raparelli V, Westphal C, Dworatzek E, Petrov G, Kararigas G, Regitz-Zagrosek V. Reduction of apoptosis and preservation of mitochondrial integrity under ischemia/reperfusion injury is mediated by estrogen receptor $\beta$. Biol Sex Differ 2016; 7(1): 53.

14. Dong $Y$, Undyala VV, Przyklenk $K$. Inhibition of mitochondrial fission as a molecular target for cardioprotection: critical importance of the timing of treatment. Basic Res Cardiol 2016; 111(5): 59.

15. Su F, Myers VD, Knezevic T, Wang J, Gao E, Madesh M, Tahrir FG, Gupta MK, Gordon J, Rabinowitz J. Bcl-2associated athanogene 3 protects the heart from ischemia/reperfusion injury. Jci Insight 2016; 1(19): e90931.

16. Wang $Y$, Zhang $H$, Chai $F$, Liu X, Berk $M$. The effects of escitalopram on myocardial apoptosis and the expression of Bax and Bcl-2 during myocardial ischemia/reperfusion in a model of rats with depression. BMC Psychiatry 2014; 14(1): 349.

17. Liu Z, Chen JM, Huang H, Kuznicki M, Zheng S, Sun W, Quan N, Wang L, Yang H, Guo HM. The protective effect of trimetazidine on myocardial ischemia/reperfusion injury through activating AMPK and ERK signaling pathway. Metabolism 2015; 65(3): 122-130.

18. Thomas CJ, Lim NR, Kedikaetswe A, Yeap YY, Woodman OL, Ng DC, May CN. Evidence that the MEK/ERK but not the PI3K/Akt pathway is required for protection from myocardial ischemia-reperfusion injury by 3',4'-dihydroxyflavonol. Euro J Pharmacol 2015; 758: 53-59.

19. Lin K, Fang S, Cai B, Huang $X$, Zhang $X$, Lu Y, Zhang $W$, Wei E. ERK/Egr-1 signaling pathway is involved in CysLT2 receptor-mediated IL-8 production in HEK293 cells. Euro J Cell Biol 2014; 93(7): 278-288. 